\title{
Biomechanical Analysis of Pedicle Screw Instrumentation in a Thoracic Scoliosis Spine Finite Element Model
}

\author{
Young Eun Kim, ${ }^{*}$, Choonki Lee ${ }^{2}$ and Soo Won Chae ${ }^{3}$ \\ ${ }^{I}$ Dept. of Mechanical Engineering, Dankook University, 126 Jukjeon-dong, Suji-gu, Yongin-si Gyeonggi-do, 448-701, \\ Korea \\ ${ }^{2}$ Dept. of Orthopedic Surgery, Seoul National University Hospital, 101 Daehak-ro, Jongro-gu, Seoul, 110-744, Korea \\ ${ }^{3}$ Dept. of Mechanical Engineering, Korea University, 5 Anam-dong, Seongbuk-gu, Seoul, 136-713, Korea
}

\begin{abstract}
In this study, the important kinematic and post-operative changes in the spinal column and rib cage after corrective surgery of scoliosis using the pedicle screw and rod derotation method was determined using a finite element scoliosis spine model, and the optimized instrumented levels for scoliosis correction was determined. A three dimensional FE scoliosis model was reconstructed by translation and rotation of a normal spinal column model using geometric mapping with x-ray and CT. By changing the fusion levels in the calculations, postoperative changes like Cobb angle, apical vertebrae axial rotation (AVAR), thoracic kyphosis, and rib hump were qualitatively analyzed. In the analysis of operative kinematics, the decrease in Cobb angle was most prominent in distraction than in deroation. Applying only the rod derotation was not effective in decreasing the Cobb angle and just caused an increase in the AVAR and rib hump. In the operative simulation, co-action of distraction and translation during rod insertion had a major impact on decreasing the Cobb angle and maintaining kyphosis. For the rod rotation, a decrease in the Cobb angle was obtained; however, this was accompanied by a simultaneous increase in the AVAR and rib hump. When the most extended instrumentation range with a $60^{\circ}$ rod rotation was used the Cobb angle was decreased in half, but an increase in the rib hump and AVAR also occurred. The optimum fusion level was found to be one level less than the inflection position of the thoracic spine curvature.
\end{abstract}

Keyword: Scoliosis, FE model, Fusion level, Post-operative changes, King-Moe type II.

\section{INTRODUCTION}

Surgical correction of scoliosis became a rather popular treatment after the Harrington rod instrumentation was developed. Various improved multisegmental instrumental technologies, such as the Luque sublaminar wire and CotrelDubousset instrumentation, in scoliosis correction have been further introduced to overcome the problems associated with the Harrington instrumentation including hook dislodgement, high pseudarthrosis rates and flatback syndrome. However, significant volumetric spinal canal intrusion may occur when using those instruments. Since the development and use of pedicle screws in the correction of idiopathic scoliosis surgery, many advantages of pedicle screw constructs for scoliosis and related deformity corrections have been documented over the last decade [1].

Surgical correction of scoliosis brings the asymmetries in the trunk back to normal shape and also prevents lumbago and weakening of the pulmonary functions. Therefore, simultaneous deliberations of the rib cage transformation and the compensative spinal bending curvature, which are all accompanied by surgical correction, should be carried out in order to produce sound surgical outcomes. As previously proven by other studies, there exists a coupled mechanism

*Address correspondence to this author at the Department Dept. of Mechanical Engineering, Dankook University, 126 Jukjeon-dong, Suji-gu, Yongin-si Gyeonggi-do, 448-701, Korea; Fax: +82-31-8005-4004; Tel: +8231-8005-3498; Email: yekim@dankook.ac.kr,yekim@dku.edu between the spine and rib cage. Since cosmetic improvement in post operation is also an important factor, changes in both the height of the rib hump and the rib cage shape, and changes in the shoulder level should also be under consideration in the surgical process.

Most scoliosis surgeries are performed by utilizing instrumentations for spinal fixation through the posterior approach. The induction of three dimensional deformations of the vertebrae with such instrumentation is, however, accompanied by many difficulties since only posterior parts are exposed during the operation. In addition, a better understanding of the factors that affect the surgical outcome prior to surgery is necessary in order to resolve post operational problems often found in the final corrected configuration of the patient.

Most of the previous studies that have examined surgical correction of scoliosis just report on the reconstruction scheme of the deformed shape during the diagnostic preoperational process, the degree of corrections that can be obtained with various surgical instrumentations and comparative results on the effects of different fixation methods. Therefore, an analytical approach that can resolve the problems of idiopathic scoliosis from a structural point of view is still needed. In this analysis, a detailed scoilotic finite element spine model that includes the rib cage and clavicle was developed. Furthermore, this model was used to analyze the biomechanical effects when applying the pedicle screw surgical instrumentation. 


\section{MATERIAL AND METHOD}

\subsection{Skeletal Model Development}

A three dimensional skeletal model was reconstructed from surface data [2] of the vertebrae, pelvis, clavicle, and scapula. These skeletal parts were modeled as rigid bodies by keeping their morphologies and they were constrained by ligaments and intervertebral discs. A kinematic joint element with six degrees of freedom was adopted to model the intervertebral disc and proper material properties were assigned so that the nonlinear behavior of the disc was accurately represented. Each ligament was precisely placed in the model based on the anatomical data set. The sigmoid shaped force-deformation characteristics of the ligament were modeled by piecewise linear curve fittings. Various material characteristics were employed with the kind and locations of the ligaments. The contact phenomena of facet joints were included by introducing the sliding interface definitions. The constructed spinal column model was then validated by comparing with in-vitro test results $[3,4]$.

Clavicles and scapulae were added to the model and they were articulated with ligaments to the rib cage, which was modeled by beam elements. The sternum was modeled by shell elements and connected to the ribs by cartilage beam elements. The costovertebral joints between vertebrae and ribs were formed by 6 DOF spring elements. The mechanical characteristics of the joint spring elements were adopted from existing experimental measurements [5] and other material properties of the model were adapted from the $\mathrm{H}$ Model data base [6]. By utilizing this normal skeletal model, a three-dimensional model was built to represent scoliosis patient. Anterior-posterior and lateral radiographic images of a twelve years old King-Moe type II patient were used. 12 anatomically representative points in the spinal column were digitized with respect to the center of a line connecting two protrusions in the vertebral pedicles, which was used as an origin axis. The coordinate values of these 12 points were used to determine the amount of deformation and axial rotation of each vertebra from the normal state. The geometric mapping was performed by a specially designed program [7]. Because of the difficulties in precisely reading the location of the pedicle from the X-radiographic images, the obtained changes in axial rotations of each vertebra were not smooth along the spinal column. Thus, the process was modified by applying a spline curve fitting to produce smooth and continuous transitions in the axial rotation between adjacent vertebrae. Articulating gap differences between the fact joints contact surfaces due to that transformation procedure were minimized by modifying the geometry of the pedicles. Meanwhile, the deformed shape of the rib cage was modeled on the basis of both CT and Xradiographic images. The overall size of the model was scaled to compensate the overall differences with the patient. Fig. (1) shows the finite element skeletal model of the normal and scoliosis models developed in this study. Due to the presence of scoliosis, changes in the mechanical properties of intervertebral discs were expected. A correction of the mechanical properties was attempted by correlating the analytic results from the model with the spinal shape changes of the patient during the voluntary lateral bending motion.

\subsection{Procedure of Surgical Simulation}

The operation began with the insertion of a pedicle screw on the concave side of each corresponding spinal segment with the prone position of the patient. The rod was then attached to the screws followed by derotation of the rod. The shape of the rod was determined by adopting the target configuration of the spine rather than the thoracic kyphotic curvature of the patient in the original state. In the case of the patient described in this study, instrumentation was applied from the upper neutral vertebra (T5) to the lower neutral vertebra (T11) shown at the standing AP view of the Xradiographic image. Accordingly, the first simulation was carried out with the same instrumentation level as used in the operation. Therefore, the rod shape was analyzed from the post operative $\mathrm{X}$ - radiographic image and the insertion of this rod into the pedicle screw, which would produce the
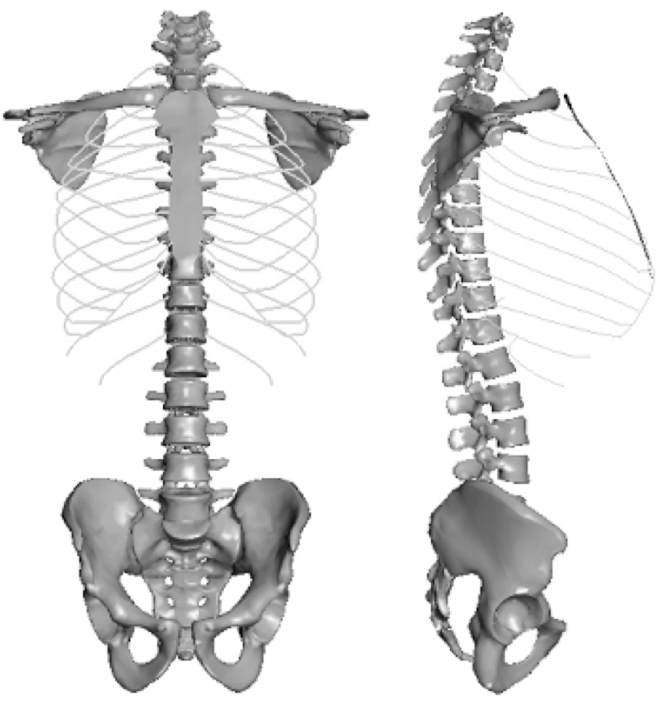

(A)
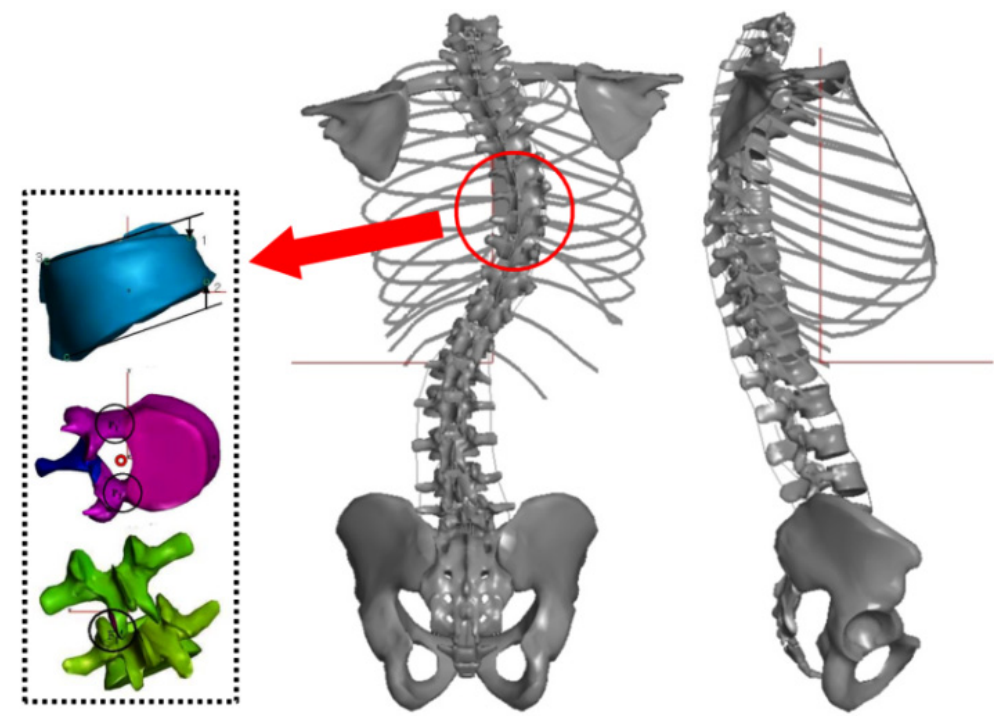

(B)

Fig. (1). Developed (A) Normal and (B) King-Moe type II scoliosis FE model. Enlarged vertebra indicates apical vertebra. 
spinal deformation, was then set up as the 1st step in the simulation. It was assumed that the scoliotic spine would deform in accordance with the rod shape during the insertion procedure. The 2 nd step in the simulation was deforming the spine during the derotation step after completion of the insertion procedure. The following boundary conditions were applied in the correction by using the pedicle screw and rod derotation. The sacrum was clamped and the axial rotation of the clavicle along the $\mathrm{z}$-axis was fixed according to the prone posture of the patient during the operation. In order to simulate the derotation of the spine with the rod, the beam elements were connected with the joint elements, positioned at the insertion points of the screws, which represent the coupling interactions between the rod and screw head. The rod elements that were axially connecting these joint elements were rotated to generate the derotation of the spine. The pivot of the derotation was performed in the direction of the local coordinate axis, which was determined by joining the most superior and inferior pedicle screws. Rotations between the pedicle screws and rod were allowed to freely occur.

The fundamental factors in operative kinematics such as distraction, translation and rod derotation were numerically performed. The changes in Cobb angle, thoracic kyphotic angle, and apical vertebral axial rotation (AVAR) were then calculated for comparative analysis and to estimate the correction effects.

The changes in the Cobb angle, thoracic kyphotic curvature, and apical vertebral axial rotation (AVAR) at each subdivided stage in the 1 st step, i.e. $25 \%, 50 \%, 75 \%$ and $100 \%$, of insertion were then calculated. During the 2 nd step of the simulation, changes in the rod derotation according to the four different rotational angles, $15^{\circ}, 30^{\circ}, 45^{\circ}$ and $60^{\circ}$, were analyzed.

After this initial test of the simulation was carried out, cases of long and short instrumentation fusions were conducted and their effects were investigated. The long instru- mentation fusion included one level above and one level below the neutral vertebra and the short instrumentation case excluded ones from each side. An identical rod curvature was used to analyze the effects of extending and reducing the instrumentation levels on scoliosis correction. In the long instrumentation case, the rod model was extended while maintaining an identical curvature to cover the T4 and T12 and the rod was connected to the vertebrae through the pedicle screws. The computation of surgical simulation using this finite element scoliosis model was performed in PamCrash 2G 2007 (ESI Group) [8].

\section{RESULTS}

A simulation of the instrumentation level of T5-11, which was the same as the actual surgical case, produced following findings. As can be seen from Fig. (2), which displays the numerical simulation results of the 1st step, when the rod was joined to the screws for the derotation operation, the Cobb angle was reduced by $30.5 \%$, i.e. from 42.26 degree to 29.39 degree, while the AVAR increased by 4.12 degrees. Fig. (3) shows the changes in the scoliotic spinal shape accompanied by the derotation process, which is the 2nd step of the operation. The Cobb angle decreased but the AVAR increased as the derotation step proceeded. A rise of the rib hump, which was in agreement with the increase in AVAR, was also observed during this process, as shown in Fig. (4). In general, the decrease of the Cobb angle in the coronal plane was larger during the rod insertion process (1st step) than during rod derotation (2nd step). A slight increase in kyphosis and the amount of AVAR during the 1st step of rod insertion was also observed in the simulation. In the early stage of rod derotation, no significant changes were observed; however, a decrease in the Cobb angle and increase in the AVAR was observed as well as a rise in the rib hump when a derotation angle of more than 30 degrees was applied.

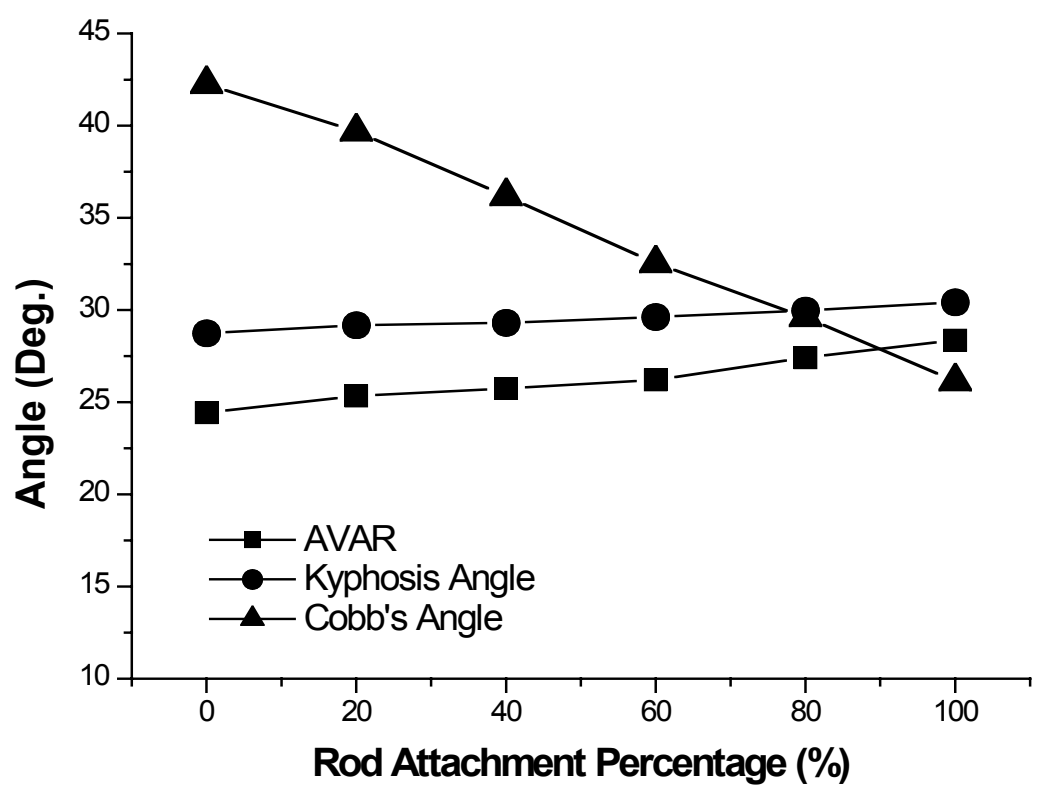

Fig. (2). Major kinetic changes during the rod attachment procedure. 


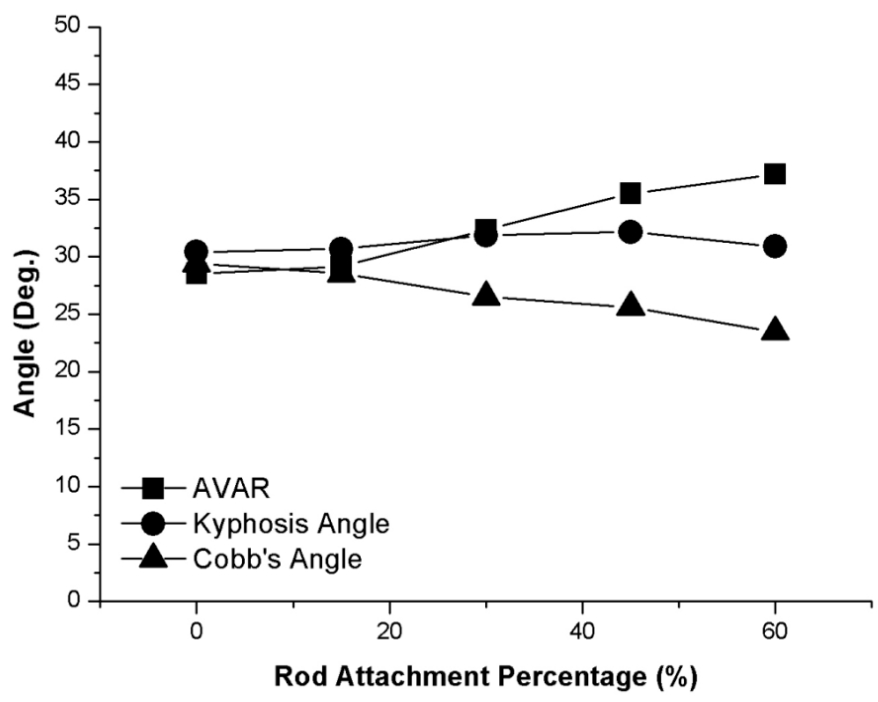

Fig. (3). Major kinetic changes during the rod derotation procedure.

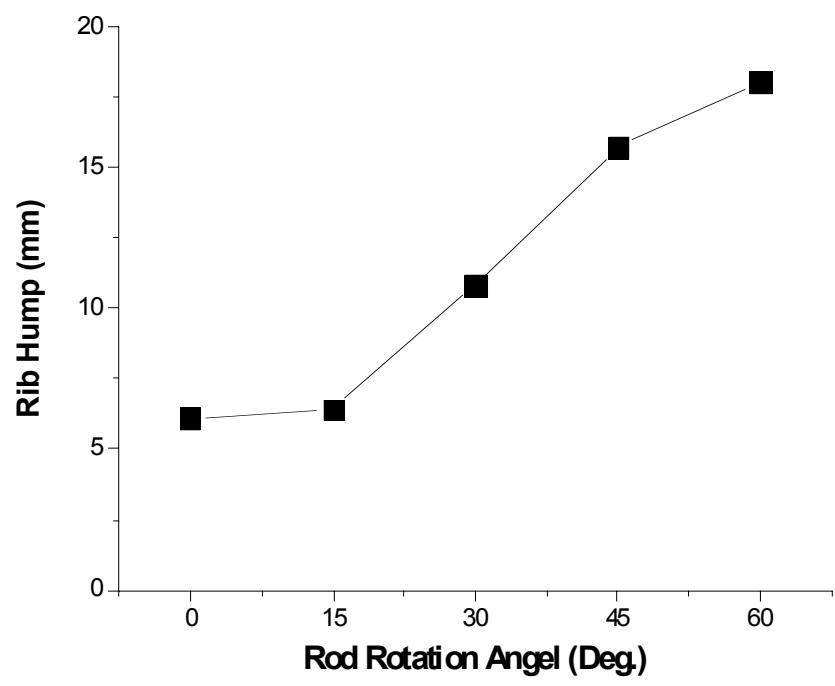

Fig. (4). Rib hump variation during the derotation procedure for the instrumentation level of T5-11.

An extension or reduction in the instrumentation level produced noticeable postoperative differences. Compared to the instrumentation level of T5-11, a short fixation resulted in a relatively small rib hump and kyposis angle, whereas a relatively small AVAR and Cobb angle were generated in the long fixation during the rod insertion step, as shown in Table 1. The fixation of T4-T12 corrected the Cobb angle by 26.1 degrees, which produced a higher correctional effect than the T5-T11 fixation. The correctional effect was further improved after the rod derotation step, which decreased the Cobb angle by 14.7 when a 60 degree rod derotation step was used. When the minimum instrumentation level was chosen as T6-T10 fixation, the Cobb angle decreased by 29.4 degrees during the rod insertion step, which was similar to the T5-T11 fixation case. However, the Cobb angle decreased to 24.2 degree after the 60 degree rod derotation step, which slightly reduced the correction effect.
Correction of the Cobb angle with different instrumentation levels was influenced more by the rod insertion step than by the rod derotation step, and the extended fixation level showed more correctional effect. The change in rib hump was proportional to the level of fixation. The change in rib hump was $35.8 \mathrm{~mm}$ with T4-12 fixation and $12.4 \mathrm{~mm}$ with T6-10 after the 60 degree rod derotation step. Fig. (5) shows the simulated results of the corrected scoliotic spines when the levels between the neutral vertebrae, the extended, and the reduced were fixed.

\section{DISCUSSION}

Based on the simulations of scoliosis correction conducted in this study, a larger change was observed during the process of rod insertion into the pedicle screw (step 1) than during the derotation step (step 2). In particular, the Cobb angle changed by $68.5 \%$ during just the insertion step 
Table 1. Major Kinetic Change According to the Instrumentation Level

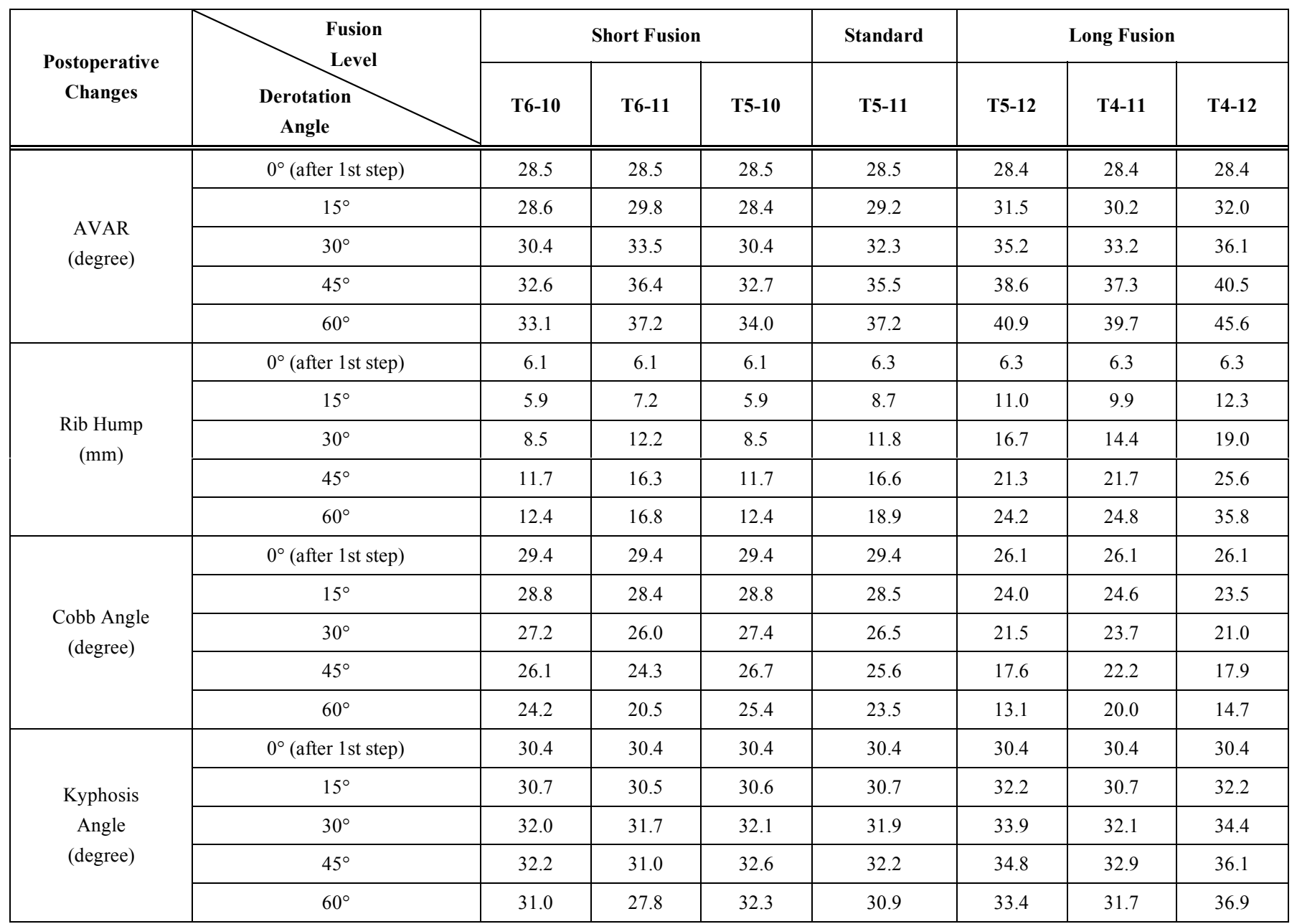

of the correction process. Stoke et al. [9] reported that lateral deformation turned into kyphotic deformation and the large decrease in the Cobb angle was then induced by rotating the rod from 90 degree to 50 degree. However, the co-occurrence of translation and elongation were not taken into account as well as the fact that model used in that study just consisted of six spinal motion segments. Compared to this finding, Poulin et al. [10] showed that a significant correction of scoliosis occurred from the insertion of the rod into the screws and hooks when a CD instrument operation was applied. Their analysis showed the synchronous production of translation and elongation, which resulted in a significant amount of scoliotic correction. During the rod insertion process in the actual operation, the rod is normally prepared with a curvature that is less than the scoliotic angle. Thus, the rod insertion process is presumed to provide rotation, translation, and elongation to the vertebrae and this phenomenon was numerically verified in this study.

Excessive rod derotation after insertion produced a decrease in the scoliotic angle but also caused too much secondary deformation such as an increase in the rib hump and rotation of the vertebrae. Different results on the degree of vertebral rotation with rod derotation have been reported: Pallock et al. [11] reported that a 30 degree change in rotational was obtained by a CD hook but Lenke et al. [12] reported just an 11 degree improvement. In contrast to these findings, Gardner-Morse and Stoke [13] reported a degradation of 8 degrees in an FEM study and a 2 degree decrease in AVAR after the rod derotation step in an intra operation performed by Lee et al. [14]. In this study, the AVAR was shown to similarly degrade with an increase in rod derotation. This analytic outcome indicates that the part of the rib cage connected on the concave side of thoracic spine played a role in supporting the wall and thus the other side of the rib cage on the convex side of thoracic spine was further pushed back, which resulted in the rise of rib hump.

The traditional fusion selection method recommended by Harrington was to fuse one above and one below the neutral vertebrae. Therefore, the fusion was performed between T5T11 for the patient used in this study. It has been believed that a short fusion holds a weak correctional force and thus can induce a secondary increase in the scoliotic angle after operation. However, the simulation results indicate that fusion with one less on each side of the neutral vertebra could minimize the change in rib hump and was the most effective in correcting the scoliotic angle. In addition, the lowest repulsive force was observed at the intervertebral disc located right under the fused vertebra and this scenario had the lowest possibility of secondary disc degeneration. This phenomenon occurred because the position of the neutral 

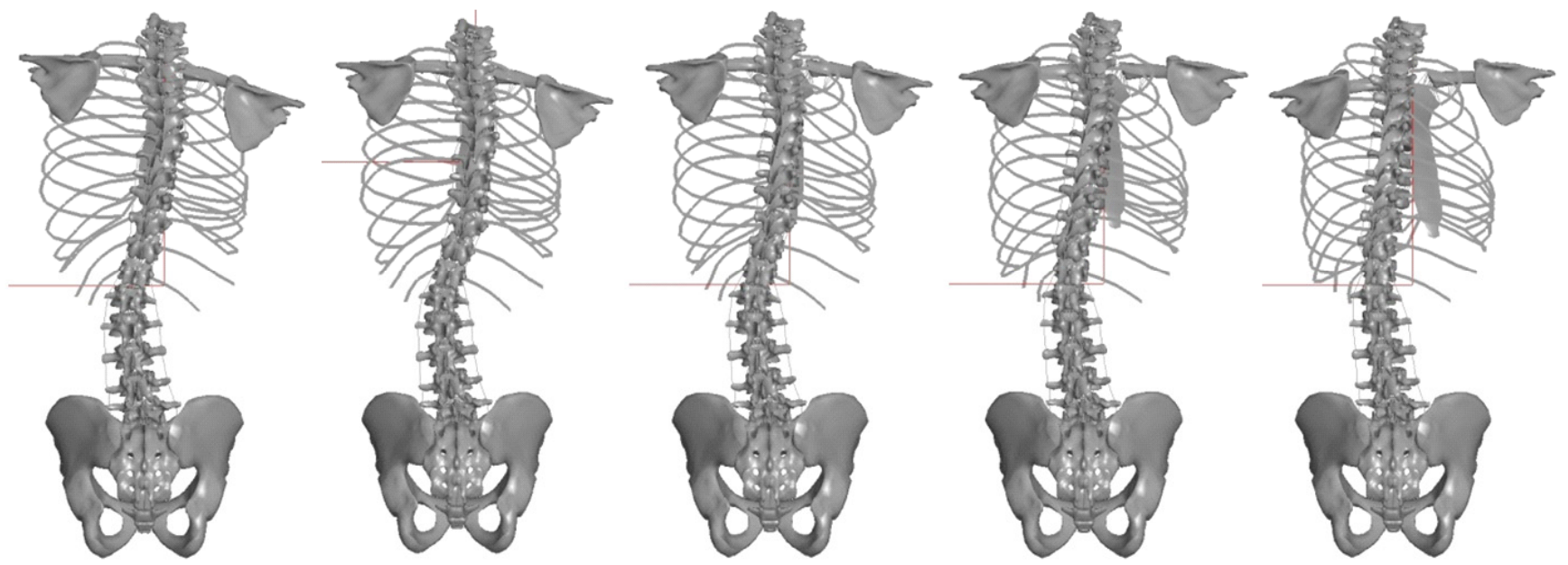

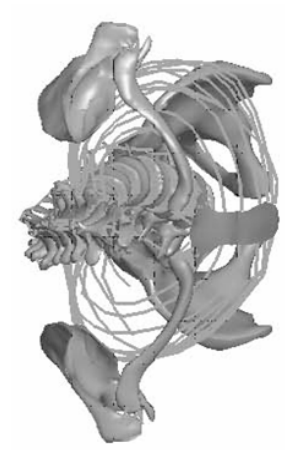

T6-T10

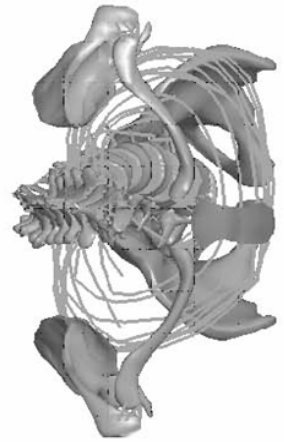

$\mathrm{T} 5-\mathrm{T} 10$

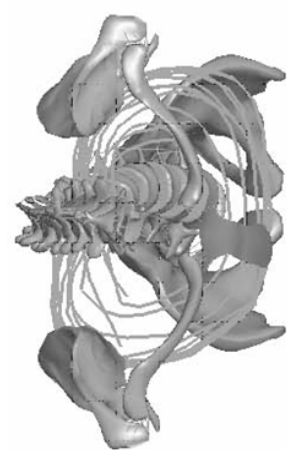

$\mathrm{T} 5-\mathrm{T} 11$

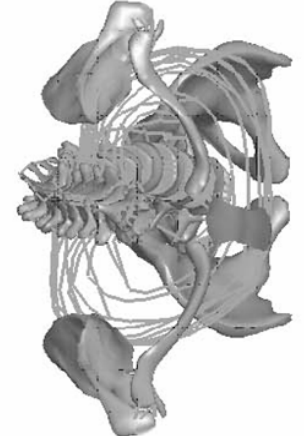

$\mathrm{T} 5-\mathrm{T} 12$

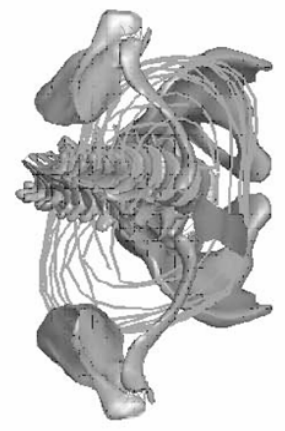

$\mathrm{T} 4-\mathrm{T} 12$

Fig. (5). Final correction shapes of the scoliotic spine for different instrumentation levels.

vertebra changed just prior to rod derotation, which was due to the considerable amount of correction in the scoliotic spine that had already occurred during the rod insertion process. Additional analysis with different scoliotic curvatures in comparison with clinical results is planned for further validation of this finding. The movable motion of the segments in the case of the main curvature in the lumbar spine needs to be maintained in this analysis and it is expected to deliver very meaningful results if the analysis is accompanied by the development of a corresponding model. If the fusion level was extended, however the scoliotic correction remarkably increased with rod derotation, which indicates that the pedicle screw fixation with rod derotation would bring more effective correction than just rod derotation alone even though a shorter fixation level was chosen for comparison to the case with a conventional Harrington instrument. It was also found that an excessive extended fixation level and rod derotation could result in a secondary increase in the rib hump and increase in the scoliotic curvature after operation.

The analysis used in this study might have the following limitations in regards to clinical applications. The skeletal muscles were not included in the model, which may have attributed to the possible over estimation of trunk deformation during rod rotation. It was also not possible to predict changes in the secondary compensation curve due to the neural control. Skeletal muscles will subsequently be included in the model to analyze its effects. The sliding of the rod along its axial direction between the screws and rod during the derotation process was not adequately taken into account in the analysis and thus a smaller amount of rod rotation was applied in the simulation than is actually used during operation. Nevertheless, the results obtained from the simulation in this study have important implications that should be considered prior to operation: no definitive cause for the increase in the rib hump after operation has been identified and no reason for the possible secondary deformation with the case of extended or reduced fixation levels have been established.

From this analysis, we found that preparing the rod with the appropriate curvature and its insertion together with a proper amount of rod rotation by considering the changes in rib hump is more important than just performing excessive derotation for scoliotic correction. Furthermore, the fixation level needs to be reduced in order to achieve an overall balance of spinal deformation rather than extended for correcting the scoliotic angle. It was also found that a short fusion, which is similar to the one used in the anterior-side approach operation, could be adopted even for the posteriorside approach operation if a strong fixation force is accom- 
panied like that from the pedicle screw fixation and rod derotation. For the case of scoliotic correction with derotation, an inappropriate amount of rod rotation results in a secondary post-operational deformation due to the connection between the rib cage and vertebrae.

\section{CONCLUSION}

In this study, a finite element model of the spinal system of a King-Moe type II patient was developed and a scoliosis surgical simulation with segmental pedicle screw fixation was performed. The results obtained from the simulation were compared to the post operational outcomes and was shown to qualitatively predict surgical effectiveness. However, this model did not include muscular imbalance and neurological control, which might lead to an over estimated amount of trunk deformation during the rod derotation process. Consequently, further steps of adding additional features to the model will be necessary to improve the accuracy of the surgical simulation. When this is complete, this preoperational simulation would be a useful tool for selecting appropriate fusion levels and surgical measures as well as predicting post-operational deformation. This model is currently being modified to investigate different Idiopathic scoliosis patients.

\section{ACKNOWLEDGEMENT}

This research was supported by Basic Science Research Program through the National Research Foundation of Korea (NRF) funded by the Ministry of Education, Science and Technology (2009-0063176).

\section{REFERENCES}

[1] Lenke LG, Kuklo TR, Ondra S, Polly D. Rational behind the current state-of-art treatment of scoliosis (in the pedicle era). Spine 2006; 33: 1051-4.

[2] Digimation premier Anatomy collection. Orlando: Digimation Inc. 2005.

[3] Yamamoto I, Panjabi MM, Crisco T. Three-dimensional movement of the whole lumbar spine and lumbosacral joint. Spine 1989; 14: 1256-60.

[4] White III A, Panjabi M. Clinical biomechanics of the Spine. Philadelphia: Lippincott Williams \& Wilkins 1999; pp.92-122.

[5] Lemosse D, Rue OL, Diop A, Skalli W, Marec P, Lavaste F. Characterization of the mechanical behavior parameters of the costo-vertebral joint. Eur Spine J 1998; 7: 16-23.

[6] H-Spinal ${ }^{\mathrm{TM}}$ user's manual. Seoul: Hankook ESI/Hangil Engineering 1998

[7] Kim YE, Ryu HK. Development of a special program for automatic generation of scoliotic spine FE model with a normal spine model. J KSPE 2006; 23: 187-94.

[8] PAM-CRASH 2G 2007 solver manual. Paris: ESI Group 2007.

[9] Stokes IAF, Laible J. Three-dimensional simulations of scoliosis by Cotrel-Dubousset instrumentation. J Biomech 1993; 27: 177-81.

[10] Poulin F, Aubin CE, Stokes IAF, et al. Biomechanical modeling of scoliotic spine instrumentation using flexible mechanisms: Feasibility study. Ann Chir 1998; 52: 761-7.

[11] Pollock FE, Pollock FE Jr. Idiopathic scoliosis: correction of lateral and rotational deformities using the Cotrel-Dubousset spinal instrumentation system. South Med J 1990; 83: 161-74.

[12] Lenke LG, Bridwell KH, Baldus C, et al. Cotrel-Dubousset instrumentation for adolescent idiopathic scoliosis. J Bone Joint Surg 1992; 74-A: 1056-67.

[13] Gardner-Morse M, Stokes IAF. Three-Dimensional simulations of scoliosis derotation by Cotrel-Dubousset instrumentation. J Biomech 1993; 27: 177-81.

[14] LEE SM, Suk SI, Kim JH, et al. Technique of pedicle screw fixation and derotation for the improvement of rotational deformity in scoliosis surgery-derotation-screw rotataion. J Korean Spine Surg $2000 ; 7: 527-34$. 